\title{
Central syntropic effects elicited by trigeminal proprioceptive equilibrium in Alzheimer's disease: a case report
}

Vincenzo De Cicco

\begin{abstract}
Introduction: The presented patient, affected by Alzheimer's disease, underwent neuropsychological evaluation and functional magnetic resonance imaging investigation under occlusal proprioceptive un-balance and re-balance conditions. Saccadic and pupillometric video-oculographic examinations were performed in order to detect connected trigeminal proprioceptive motor patterns able to interfere with reticular formation cerebellum functions linked to visual and procedural processes prematurely altered in Alzheimer's disease.

Case presentation: A 66-year-old Caucasian man, affected by Alzheimer's disease and with a neuropsychological evaluation issued by the Alzheimer's Evaluation Unit, underwent an electromyographic investigation of the masseter muscles in order to assess their functional balance. The patient showed a bilateral lack of all inferior molars. The extreme myoelectric asymmetry in dental occlusion suggested the rebalancing of masseter muscular functions through concurrent transcutaneous stimulation of the trigeminal nerve supramandibular and submandibular motor branches. The above-mentioned method allows detection of symmetric craniomandibular muscular relation that can be kept constant through the use of a cusp bite modeled on the inferior dental arch, called orthotic-syntropic bite. A few days later, the patient underwent a new neuropsychological investigation, together with a functional magnetic resonance imaging study, and saccadic, pupillometric video-oculographic examinations in occlusal un-balance and re-balance conditions.
\end{abstract}

Conclusions: Comparative data analysis has shown that a re-balanced occlusal condition can improve a patient's cognitive-attentive functions. Moreover, the saccadic and pupillometric video-oculographic investigations have proven useful both in analyzing reticulo-cerebellar subcortical systems, prematurely altered in Alzheimer's disease, and in implementing neurological evaluations.

\section{Introduction}

An increasing amount of evidence has pointed out the effects generated in aged rats by the loss of molar teeth, with reduction of spatial memory, acetylcholine release from the parietal cortex [1] and alteration of the septohippocampal cholinergic system [2], while Yamazaki et al. [3] have verified that the number of extracted teeth was directly proportional to the loss of spatial memory and to the reduction of trkB messenger ribonucleic acid (mRNA) levels. Furthermore, epidemiological surveys and crosssectional studies in subjects ranging from 50 to 80 years old have demonstrated that reduced chewing ability or

Correspondence: vincenzodecicco4@virgilio.it

Department of Oral Science, University 'G.D'Annunzio', via dei Vestini, 31, Chieti 66100, Italy dysfunctional teeth induced senile processes or degeneration of hippocampal neurons, with a decrease of cognitive function and learning effect $[4,5]$. On the basis of these findings, studies have been carried out to verify whether the rebalance of myoelectric asymmetries in dental occlusion could modify cognitive-attentive parameters, even in a subject affected by Alzheimer's disease (AD). In line with the above-mentioned findings, a case report random selection has envisaged only the bilateral lack of all inferior molars and a neuropsychological evaluation of average seriousness. Moreover, computerized saccadic and pupillometric video-oculographic evaluations were performed for their cognitive-attentive and mnemonic nature [6]. These examinations have also allowed for the analysis of the response of reticular formation and cerebellum 
nuclei connected with visio-motor and procedural processes, prematurely altered in $\mathrm{AD}[7,8]$.

\section{Case presentation}

The patient was a 66-year-old Caucasian man, who presented for a neuropsychological evaluation performed at the Centre of Alzheimer's Evaluation Unit (AEU), with a Mini-Mental State Examination (MMSE) value of 18/30. The neuropsychological final report, as authentically quoted and faithfully pursuant to the description of the AEU doctor, related the following: 'The present condition offers a picture of poor collaboration on the part of the patient who shows attention loss while performing the test and needs to be reminded about the given indications. Remarkable language worsening with reduced capacity of expression and comprehension that limit his autonomy. Worsening of executive functions with difficulty in planning and performing even simple activities, difficulty in solving problems. This picture defines a significant loss in instrumental activities for which assistance is needed. Treatment with anticholinesterasics and antioxidants must be continued' (Table 1). For the evaluation of occlusal muscle activity, bilateral electromyography (EMG) of the masseter muscle was recorded using surface $\mathrm{Ag} / \mathrm{AgCl}$ electrodes. In accordance with dental diagnostic protocols $[9,10]$, a preliminary evaluation of the patient's myoelectric activity in habitual dental

Table 1 Neuropsychological values

\begin{tabular}{|c|c|c|c|}
\hline Category & Subcategory & $\begin{array}{l}\text { Occlusal } \\
\text { habitual }\end{array}$ & $\begin{array}{l}\text { Orthotic-syntropic } \\
\text { bite }\end{array}$ \\
\hline \multirow[t]{2}{*}{ Orientation } & Spatial & $3 / 5$ & $5 / 5$ \\
\hline & Temporal & $2 / 5$ & $4 / 5$ \\
\hline \multirow[t]{2}{*}{ Memory } & $\begin{array}{l}\text { Immediate Rey } \\
\text { test }\end{array}$ & $11 / 75$ & $15 / 75$ \\
\hline & $\begin{array}{l}\text { Deferred Rey } \\
\text { test }\end{array}$ & $3 / 157 / 15$ & \\
\hline Reasoning & Raven matrices & $20 / 36$ & $22 / 36$ \\
\hline \multirow[t]{3}{*}{ Language } & Token test & $18 / 36$ & $21 / 36$ \\
\hline & $\begin{array}{l}\text { Verbal flux per } \\
\text { category }\end{array}$ & 8 & 9 \\
\hline & $\begin{array}{l}\text { Verbal flux per } \\
\text { letter }\end{array}$ & 14 & 13 \\
\hline Attention & $\begin{array}{l}\text { Immediate visual } \\
\text { memory }\end{array}$ & $14 / 22$ & $18 / 22$ \\
\hline \multirow[t]{2}{*}{ Apraxias } & Ideational & $17 / 20$ & $18 / 20$ \\
\hline & Constructional & $12 / 20$ & $16 / 20$ \\
\hline \multicolumn{2}{|l|}{$\begin{array}{l}\text { Instrumental Activities } \\
\text { of Daily Living (IADL) }\end{array}$} & $4 / 8$ & $6 / 8$ \\
\hline \multicolumn{2}{|l|}{$\begin{array}{l}\text { Activities of Daily } \\
\text { Living (ADL) }\end{array}$} & $4 / 6$ & $6 / 6$ \\
\hline \multicolumn{2}{|l|}{$\begin{array}{l}\text { Mini-Mental State } \\
\text { Examination (MMSE) }\end{array}$} & $18 / 30$ & $23 / 30$ \\
\hline
\end{tabular}

occlusion was performed via electromyography of the muscles in order to assess their functional balance, as the patient showed a bilateral lack of all molars, second premolars and the right medial incisor. Registered values showed a remarkable functional asymmetry of the masseter muscles: $10 \mathrm{mV}$ for the left masseter and $111 \mathrm{mV}$ for the right masseter, respectively (Figure 1). According to the expressed electromyographic values, muscular activity was symmetrized by applying a 15 -min transcutaneous stimulation of the trigeminal motor branches at low frequency for the masseter muscles and at medium frequency for the submandibular antagonist muscles. This method allowed detection of the functional trajectory of the occlusal elevator muscles and the recording of a symmetric craniomandibular relation by positioning a self-hardening material between the dental arches. The same material has been used successively to make a cusp bite modeled on the inferior dental arch, named the orthotic-syntropic bite for its particular use of electrostimulation. When the orthotic was applied, electromyographic control was repeated to verify the occlusal myoelectric balance. Substantially equal values were shown: $55 \mathrm{mV}$ for the left masseter muscle and $60 \mathrm{mV}$ for the right muscle (Figure 2).

Immediately after, saccadic, pupillometric and functional MRI (fMRI) examinations were performed, in habitual occlusion first and with the orthotic soon after; then, after a few days the patient was submitted again for neuropsychological evaluation. The saccadic videooculography system used (Figure 3) is made up of a computerized eye-tracking system with eye tracking acquisition times of under 100 milliseconds. It is able to measure on a space-time diagram the distance between the point at which the patient is being observed and the point at which the target is situated in that exact

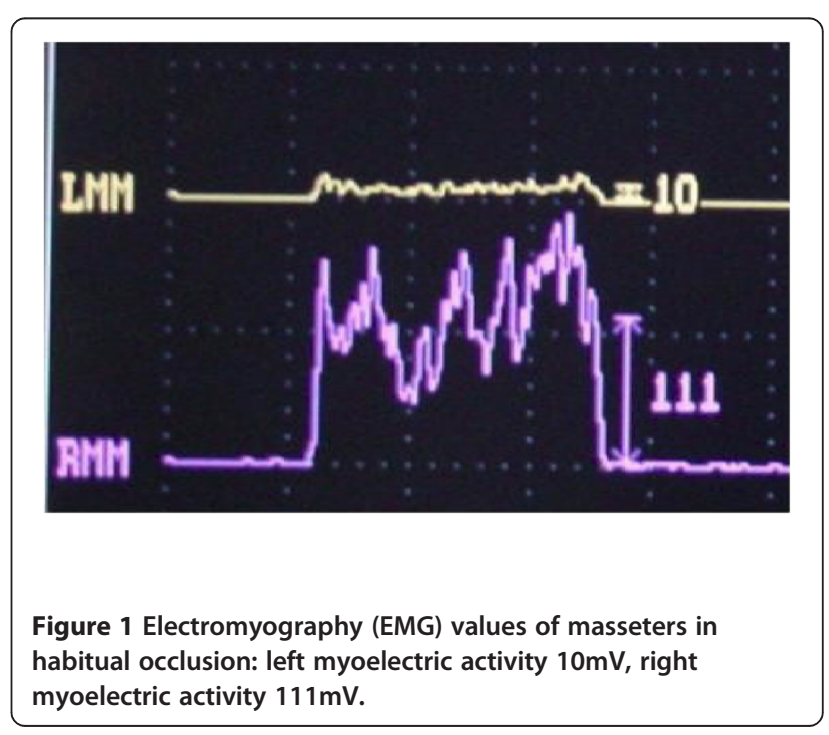




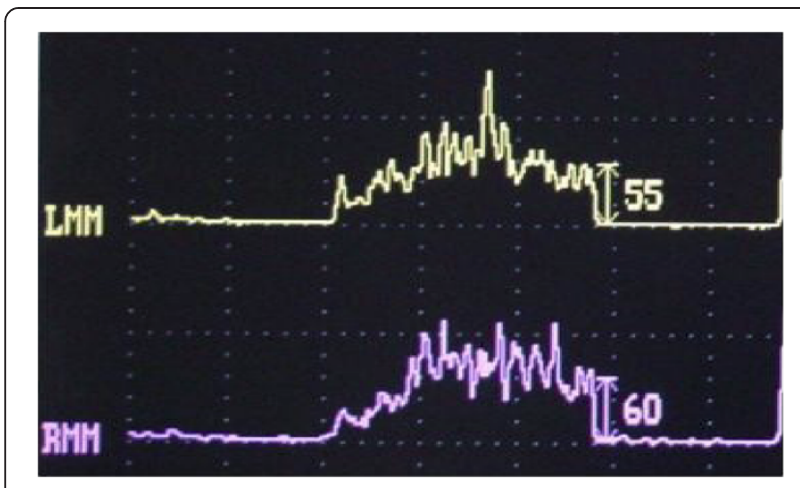

Figure 2 Electromyography (EMG) values of masseters in habitual occlusion: left myoelectric activity $55 \mathrm{mV}$, right myoelectric activity $60 \mathrm{mV}$.

moment. The system can evaluate the target/eye coherence for 16 points, showing hypometric and hypermetric mistakes with drifts of different extension and the total visual attentive incoherence of the patient with rectangles. The saccadic test calculates variable target routes that do not allow mnemonic effects. Indeed, in the occlusal un-balance condition, the video-oculographic program recorded complete target/eye inconsistency for 12 of the 16 detection points (Figure 4), while in the rebalance condition there were only two reported mistakes (Figure 5). Pupil diameter evaluations were measured with a corneal topographer made up of a survey section with a Placido disk (24 loops), charge-coupled device (CCD)1/3 camera sensor with $56 \mathrm{~mm}$ working distance, and constant light and a chin support. The cognitive task given was a perceptive motor practognosic test,

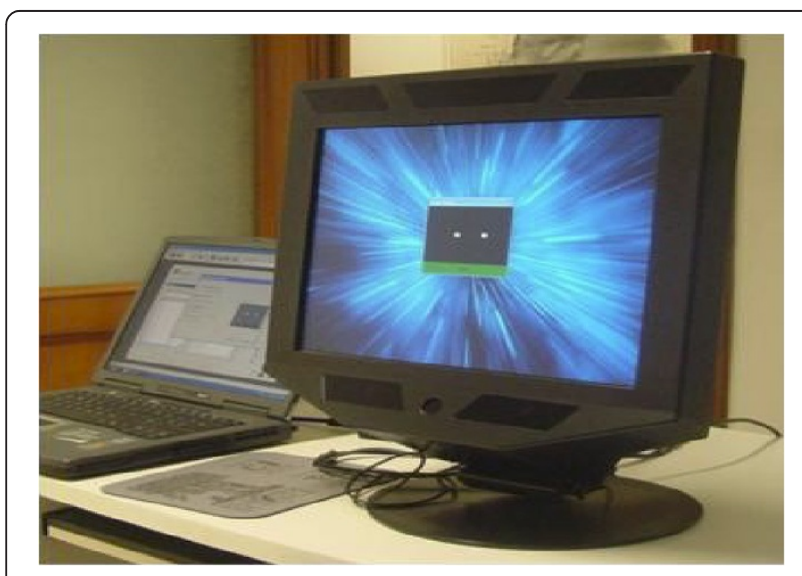

Figure 3 Video-oculographic system for saccadic test: the two white points that appear on the screen represent the fovea centralis retinae. named TanGram, made up of a puzzle of triangular, square and parallelogram-shaped geometric forms (Figure 6). The patient, who had previously had how to perform the test explained to him, had to reposition in its specific place, without visual support, an element of the puzzle taken out and put in his right hand by the operator. The adopted protocol provided the pupillary diameter measurements (first basal and then during the cognitive task) just two seconds after the beginning of site exploration in the box. Pupillometric recordings were more interesting because when the patient performed the TanGram test, his basal pupillary diameter (2.65 $\mathrm{mm}$ ) (Figure 7), which is physiologically inclined to extension, reduced by $-0.21 \mathrm{~mm}(2.44 \mathrm{~mm})$ (Figure 8 ), while in the occlusal re-balance condition a pupillometric increase of $+0.58 \mathrm{~mm}(3.14 \mathrm{~mm})$ (Figures 9 and 10) was registered, in line with what has been previously reported in the literature (Table 2). The results of fMRI performed in un-balance and re-balance conditions have been reported jointly and they precisely refer to: 'type of performed exam: direct cerebral RM. Poorly collaborative patient. No significant areas of diffusion restriction. Some foci with gliotic signs related to old vascular problems can be detected in periventricular and subcortical areas. Insignificant signal alterations in the subtentorial area. Increase of ventricular cavities width, especially of the left occipital horn and of the subarachnoid spaces with an atrophic basis. It was not possible to perform a functional study with the activation of motor areas through right hand finger tapping due to the patient's inability to perform the task correctly. After trigeminal stimulation and dental bite application, the functional study was made possible by an improved collaboration on the part of the patient, even if a correct activation of the pertinent motor area was not possible because the given orders were not performed at the same time as the acquisitions'.

Finally, a neuropsychological report, undertaken after orthotic-syntropic bite application, related the following: 'The neuropsychological evaluation evidences a higher collaboration of the patient, he is well oriented in space and shows light temporal disorientation. Minor difficulty in the comprehension of simple orders while the impediment to perform more complex tasks remains. The patient has improved short and medium term verbal memory span and his autonomy in performing instrumental activities of daily living, needing assistance only for more complex activities. Ideational slowdown remains. Improvement in executive functions capacities is evidenced, especially in planning, organization and problem solving abilities', while the MMSE values in re-balance occlusion showed significant improvement (23/30), five points higher than the previous study (18/30) in the habitual condition (Table 1). 


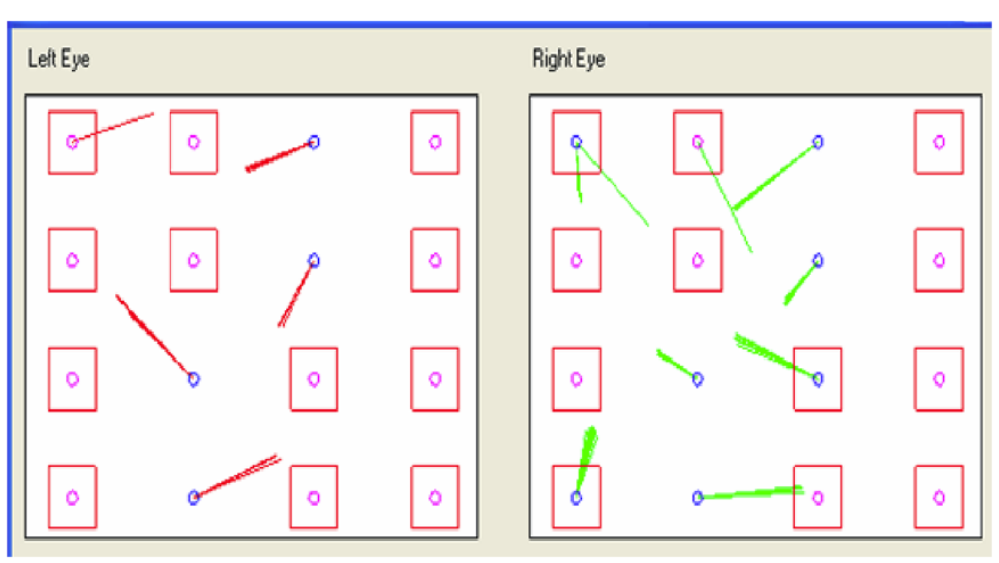

Figure 4 Video-oculographic saccadic test in habitual occlusion: 12 squares.

\section{Discussion}

The results obtained in occlusal un-balance and rebalance conditions suggest that occlusal proprioceptive asymmetries can elicit central anisotropic effects characterized by minimum order configurations and minimum cortical-subcortical functional differentiations. Even if references to the literature are only indirect at the moment, in this case report the performed tests permit us to deduce that occlusal proprioceptive re-balance in the short/medium term can alter some central functional parameters. Overall, data analysis suggests that consistent effects are seen in the visual-spatial context, in the planning and execution of organization skills, while other functions, such as ideation, reasoning and execution of complex tasks, language and grammar complexity do not display significant results. Improved collaboration on the part of the patient during neuropsychological evaluation and in fMRI execution is a very significant element to take into account. With respect to this, the pupillometric exam can contribute to our understanding of the behavioral change on the part of the patient, because pupillary diameter variation represents an unequivocal evaluative element of the cognitive control state during an evoked task and it is strictly related to locus coeruleus (LC) tonic/ phasic activity [11]. In fact, Cohen et al. have demonstrated that during task execution the anterior cingulate, orbitofrontal and prefrontal cortex stimulates LC phasic mode with norepinephrine release. This determines concomitant and immediate pupillary diameter increase, proportional to the released noradrenergic quantity [12]. A pupillometric reduction of $-0.21 \mathrm{~mm}$ registered in occlusal un-balance during the test (Figures 7 and 8 ) may be

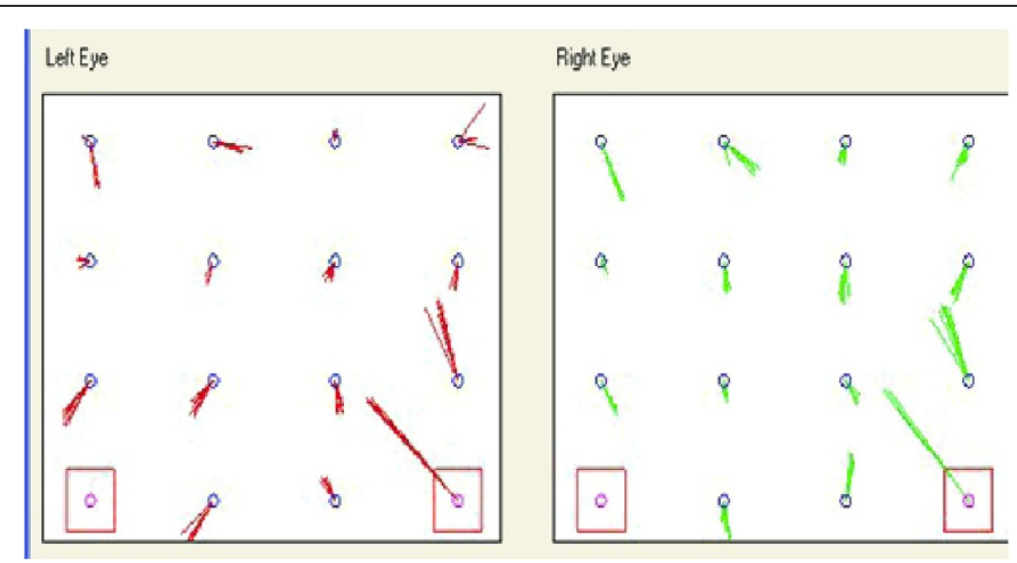

Figure 5 Video-oculographic saccadic test after orthotic-syntropic application: two squares. 


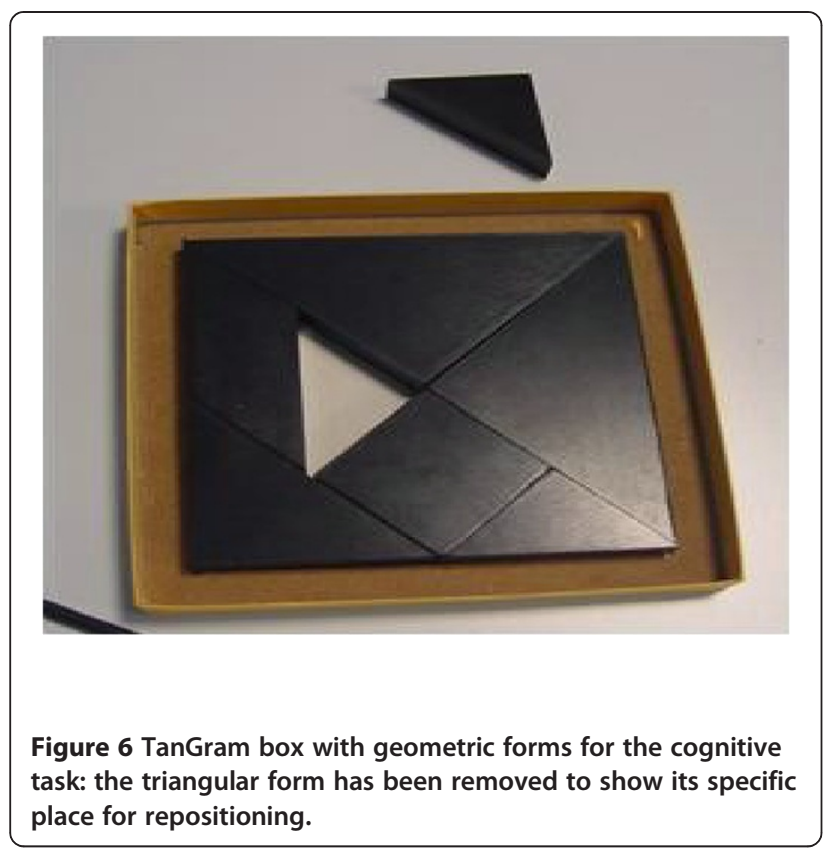

interpreted as the result of cortical strain [13], while a pupillometric increase of $+0.58 \mathrm{~mm}$ registered in occlusal re-balance, with a basal pupillometric value of $2.62 \mathrm{~mm}$ (Figures 9 and 10), is an index of unquestionable higher coerulean phasic expressivity. This is surprising because the LC is prematurely and deeply interested by AD degenerative processes [14], and it is also interesting with regard to basal reduction. Trigeminal neurophysiological mechanisms at the core of pupillometric clinical evidence cannot be exhaustively delineated at present, but some relationships among the trigeminal complex, coerulean system and reticular formation can be hypothesized. The literature mainly relates the projections and effects of the LCnorepinephrine system on trigeminal sensorimotor nuclei. Previous studies performed through anterograde and retrograde transport analysis have indicated that many of the regions that received dense inputs from the projected LC neurons, in turn, back upon these coerulei neurons [15], which are uniformly sensitive to a variety of non-noxious stimuli, including tactile, visual, auditory and taste with specific degree of activation stimulus, [16,17]. The trigeminal system is strictly connected with the LC and several works have proved that clusters of mesencephalic neuronal branches reach LC-pars compacta, which exhibit a mixture of cellular elements with trigeminal mesencephalic neurons, $[15,18]$. Couto et al. demonstrated with retrograde tract tracing using fast blue injections in spinal and principal sensory trigeminal nuclei, the presence of labeled trigeminal mesencephalic and cerulean neurons, [19]. Moreover, Panneton et al. proved trigemino-autonomic connections, using herpes simplex virus 1 (HSV-1) (strain 129), with an anterograde transneuronal transport method that LC and paragigantocellularis nuclei were also labeled [20]. Seemingly, the LC can be activated by increasing the discharge frequency of trigeminal mesencephalic neurons activated both by masseter spindle receptors due to interocclusal excessive space [21], and by the periodontal for increased occlusal charge, with glutamate release for the activation of presynaptic $\gamma$-aminobutyric acid $\left(\mathrm{GABA}_{\mathrm{A}}\right)$ receptors, on the coerulean and peri-coerulean zone [22]. These conditions,

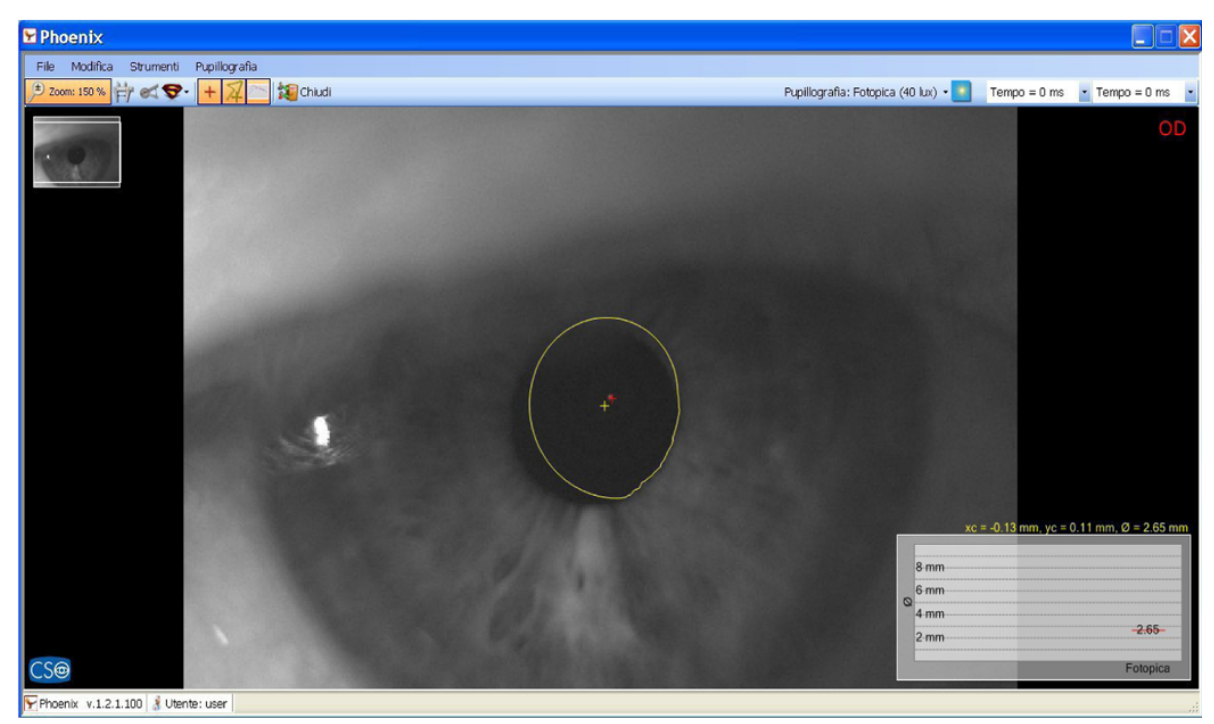

Figure 7 Basal pupillometry in habitual occlusion; pupil diameter: $2.65 \mathrm{~mm}$. 


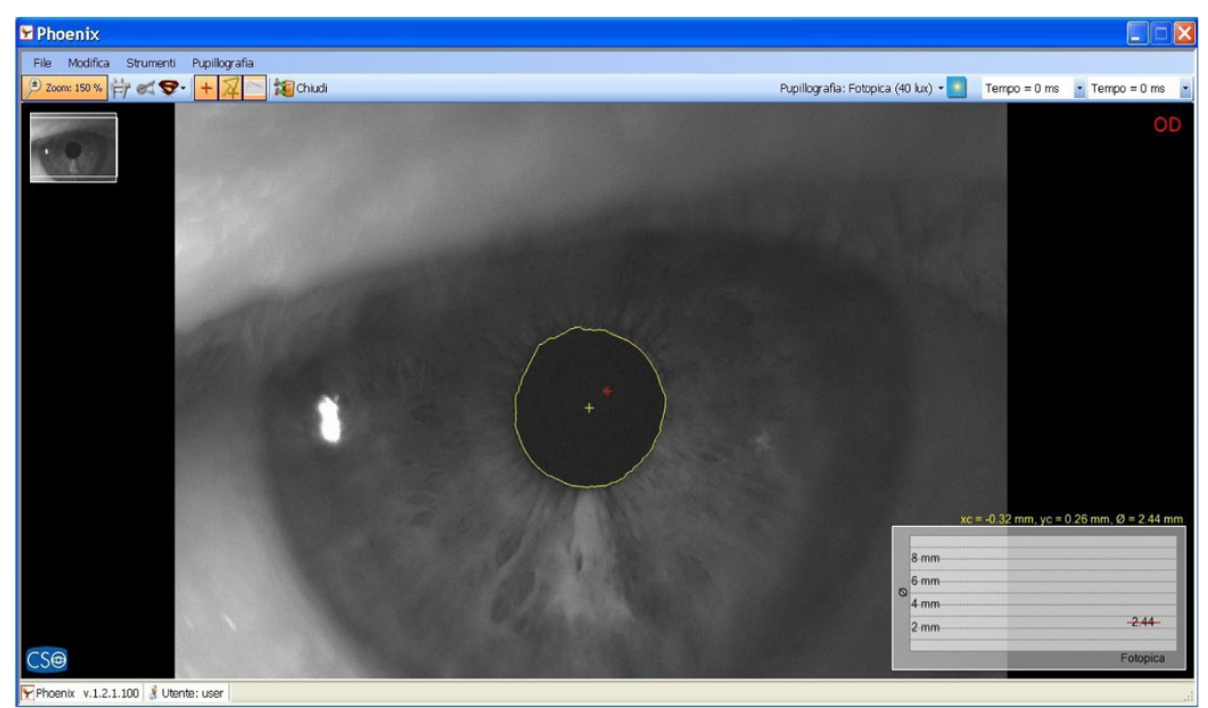

Figure 8 Pupillometry in TanGram test in habitual occlusion; pupil diameter: $2.44 \mathrm{~mm}$.

characterized by neuromotor facilitation of the mastication preferential side, are inevitably associated with contralateral functional hypoactivity of the trigeminal nerve motor and mesencephalic nuclei. Occlusal motor-proprioceptive activity probably produces a concomitant and homolateral asymmetry of LC/noradrenaline (LC-NE) system phasic modes. Specifically, we may believe that occlusal balance symmetrization can determine, in the trigeminal/LC-NE mesencephalic nucleus pathway, a coerulean activation on the hypoactive occlusal side and a concomitant contralateral reduction which, moreover, could also determine a lower galanin release, normally hyperexpressed in $\mathrm{AD}$, from noradrenergic terminations [23,24]. In fact, Hoogendijk et al. have demonstrated through the determination of $\mathrm{NE}$ and of its 3-methoxy-4-hydroxyphenylglycol (MHPG) metabolite in different brain areas that a significant reverse relationship between the number of coerulean neurons and MHPG/NE ratio both in frontal cortex and in LC can be found in subjects affected by $\mathrm{AD}$, while a significant rise of the MHPG/NE ratio indicates a consistently increased metabolism [25]. In addition to this hypothesis, the coerulean area can also be indirectly activated by the trigeminal motor nucleus. This nucleus does not have a definite nuclear delimitation but it is mixed with lateral reticular formation

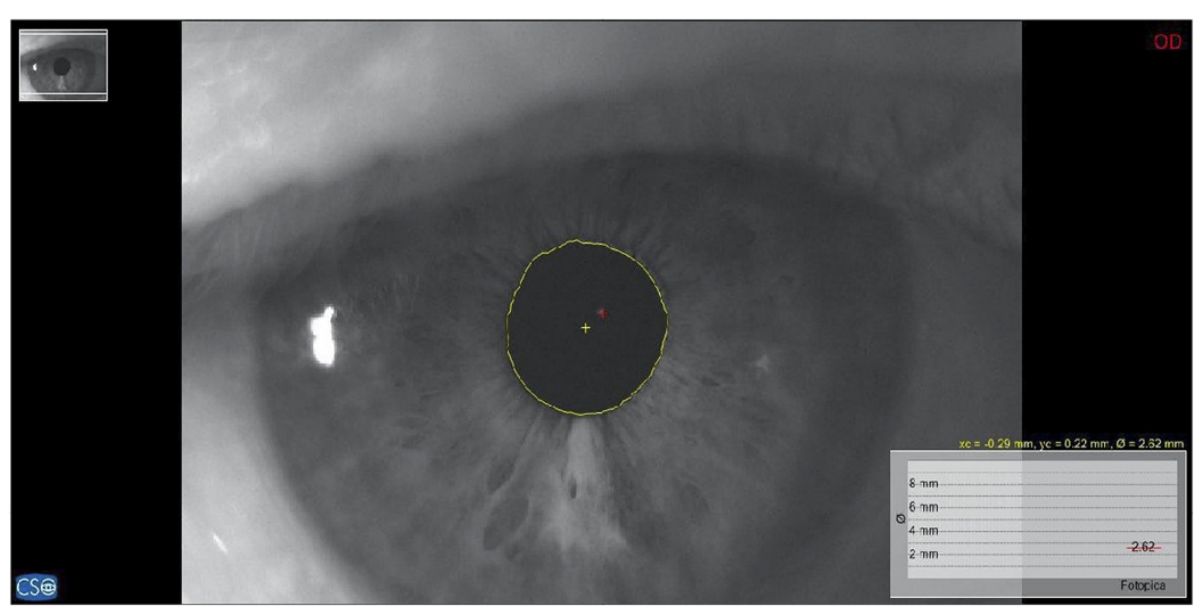

Figure 9 Basal pupillometry with orthotic-syntropic application; pupil diameter: $2.62 \mathrm{~mm}$. 


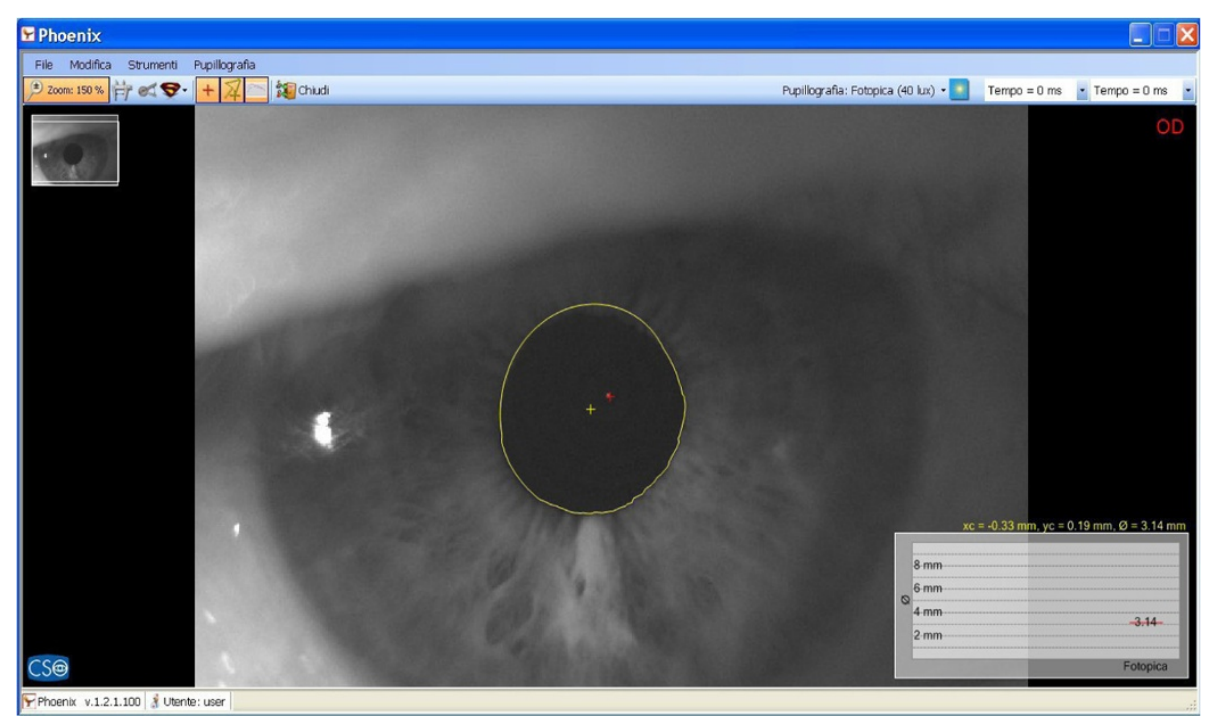

Figure 10 Pupillometry in TanGram test with orthotic-syntropic application; pupil diameter: $3.14 \mathrm{~mm}$.

(LRF) parvocellular neurons [26], and it is part of the ascending reticular activating system [27]. Presumably, neuromotor hyperactivity of the mastication preferential side elicits a concomitant asymmetric brainstem stimulation reward [28], including diffused projection catecholaminergic systems of intermediate reticular formation nuclei (IRFn). Previous research has recorded short latency hypsilateral orthodromic responses in LRF and IRFn after electrostimulation of the masseteric nerve and after passive mandible dislocations, [29]. Therefore, a hypothesis can be made that the pupillary diameter increase $(3.14 \mathrm{~mm})$ (Figure 10) registered in occlusal re-balance during the evoked task may be the result of a more effective and synchronous phasic expressivity of cerulean neurons, associated with a more suitable reduction $(2.62 \mathrm{~mm}$ ) (Figure 9) of the basal diameter. This last result further validates the functional relationships between coerulean and trigeminal systems because, on the one hand, Rajkowski et al. have demonstrated that basal pupillary diameter is strictly connected to LC tonic discharge frequency. On the other hand, Yabushita et al. have shown that the occlusal vertical dimension increase, which we obtained by orthotic syntropic application, reduces neuromuscular spindle discharge frequency of the masseter muscles [21]. The cited research also suggests that an increased inter-occlusal free space inevitably implies

Table 2 Computerized recordings of pupil diameters

\begin{tabular}{lll}
\hline & Occlusal habitual & Orthotic-syntropic bite \\
\hline Basal, $\mathrm{mm}$ & 2.65 & 2.62 \\
TanGram test, $\mathrm{mm}$ & 2.44 & 3.14 \\
\hline
\end{tabular}

extreme masseter muscle contraction during occlusion in swallowing, determining an increase of spindle and periodontal discharge frequency that hyperactivates the mesencephalic nucleus with glutamate, having depolarizing functions on cerulean neurons. The final result is an increased LC tonic activity that can be detected in the pupillary basal diameter size. The results obtained from saccadic and pupillometric tests confirm the above. Reports in the literature state that saccadic final control is developed by perfect coordination and synchrony of both prepositus hypoglossi and paramedian pontine reticular nuclei [30,31], and the ipsicerebellar fastigial contralateral to saccadic movement nuclei [8]. Fastigial nuclei can certainly be a target of occlusal asymmetric motor activity, especially of the hypofunctional factor, since the brainstem burst generator cannot produce accurate saccades without oculomotor cerebellum contribution [32]. In fact, these nuclei operate a codification of saccadic command space-time transformation through absolute functional synchrony of the contralateral (initial facilitation of the 'burst' scale) and ipsilateral (late 'burst' discharge inhibition) fastigial nuclei to saccadic movement and their diminished cooperation can determine saccadic hypo/ipermetry [33]. At present, it is not possible to confirm if the targets of occlusal asymmetry are mainly reticular nuclei, cerebellum fastigial nuclei, or both, but the radical improvement registered in the saccadic test after occlusal re-balance can be interpreted, in apparent contrast with the high stability of the neuronal systems controlling and programming it, as an index of reticulo-cerebellar functional synchrony, equal to what has been hypothesized for coerulean neuron activation modes. 


\section{Conclusions}

The results of this case report suggest that occlusal unbalance may represent an interferential pattern in some AD central functions. Pupillary dynamic and saccadic motor control examinations can be an efficient investigation tool for implementing neurologic evaluations, and identifying patterns interfering with tonic/phasic LC modes. Within the limits of this case report, further investigations are necessary in order to detect the modalities by which the muscular and periodontal proprioception may modulate the cognitive attentive activity.

\section{Consent}

Written informed consent was obtained from the patient's next of kin for publication of this manuscript and any accompanying images. A copy of the written consent is available for review by the Editor-in-Chief of this Journal.

\section{Competing interests}

The author declares that he has no competing interests.

\section{Acknowledgements}

The author sincerely thanks Enrico Cagliari for the acquisition and interpretation of the patient data regarding the fMRI.

Received: 17 October 2011 Accepted: 26 June 2012

Published: 26 June 2012

\section{References}

1. Kato T, Usami T, Noda Y, Hasegawa M, Nabeshima T: The effect of the loss of molar teeth on spatial memory and acetylcholine release from the parietal cortex in aged rats. Behav Brain Res 1997, 83:239-242.

2. Watanabe K, Onozuka M, Fujita M, Ozono S: Changes in the septohippocampal cholinergic system following removal of molar teeth in the aged SAMP8 mouse. Behav Brain Res 2002, 133:197-204.

3. Kaoruko Y, Wakabaiashi N, Kobayashi T, Suzuki T: Effect of tooth loss on spatial memory and trkB-mRNA levels in rats. Hippocampus 2008, 18: 542-547.

4. Bergdahl M, Habib R, Bergdhal J, Nyberg L, Nilsson LG: Natural teeth and cognitive function in humans. Scand J Psychol 2007, 48:557-565.

5. Ono T, Hori K, Ikebe K, Nokubi T, Nago S, Kookaburra I: Factors influencing eating ability of old in-patients in a rehabilitation hospital in Japan. Gerondontology 2003, 20:24-31.

6. Liversedge S, Findlay J: Saccadic eye movements and cognition. Trends Cognit Sci 2000, 4:6-14.

7. Inaba N, Iwamoto Y, Yoshida K: Changes in cerebellar fastigial burst activity related on saccadic gain adaptation in the monkey. Neurosci Res 2003, 46:359-368.

8. Ohtsuka $\mathrm{K}$, Noda $\mathrm{H}$ : Burst discharges of fastigial neurons in macaque monkeys are driven by vision and memory-guided saccades but not by spontaneous saccades. Neurosci Res 1992, 15:224-228.

9. Nnoaham KE, Kumbang J: Transcutaneous electrical nerve stimulation (TENS) for chronic pain. Cochrane Database Syst Rev 2008, 3:CD003222.

10. Didier H, Marchetti C, Borromeo G, Tullo V, Bussone G, Santoro F: Persistent idiopathic facial pain: multidisciplinary approach and assumption of comorbidity. Neurol Sci Supp/ 2010, 1:s189-s195.

11. Rajkoski J, Kubiak P, Aston-Jones G: Correlations between locus coeruleus (LC) neural activity, pupil diameter and behaviour in monkey support a role of LC in attention. Soc Neurosci Abstr 1993, 19:974.

12. Gilzenrat MS, Nieuwenhuis S, Jepma M, Cohen JD: Pupil diameter tracks changes in control state predicted by the adaptive gain theory of locus coeruleus function. Cogn Affect Behav Neurosci 2010, 10:252-269.

13. Granholm E, Asarnow R, Sarkin A, Dykes K: Pupillary responses index cognitive resource limitations. Psychophysiology 1996, 33:457-461.

14. Braak H, Del Tredici K: Alzheimer's pathogenesis: is there neuron-toneuron propagation? Acta Neuropathol 2011, 121:589-595.
15. Cedarbaum JM, Aghajanian GK: Afferent projections to the rat locus coeruleus as determined by a retrograde tracing technique. J Comp Neurol 1978, 178:1-16.

16. Aston-Jones G, Bloom FE: Norepinephrine containing locus coeruleus in behaving rats exibit pronounced responses to nonnoxious environmental stimuli. J Neurosci 1981, 1:887-900.

17. Sara S, Herve A: Plasticity of auditory responses of locus coeruleus neurons: studies in anesthetized rats. Soc Neurosci Abstr 1993, 19:413.

18. Luo P, Zhang J, Yang R, Pendlebury W: Neuronal circuitry and synaptica organization of trigeminal proprioceptive afferents mediating tongue movement and jaw-tongue coordination via hypoglossal premotor neurons. Eur J Neurosci 2006, 23:3269-3283.

19. Couto LB, Moroni CR, dos Reis Ferreira CM, Elias-Filho DH, Parada CA, Pela $I R$, Coimbra NC: Descriptive and functional neuroanatomy of locus coeruleus-noradrenalin-containing neurons involvement in bradykiniinduced antinociception on principal sensory trigeminal nucleus. $J$ Chem Neuroanat 2006, 32:28-45.

20. Panneton WM, McCulloch PF, Sun W: Trigemino-autonomic connections in the muskrat: the neural substrate for the diving response. Brain Res 2000, 874:48-65.

21. Yabushita T, Zeredo $\mathrm{J}$, Toda K, Soma K: Role of occlusal vertical dimension in splindle function. J Dent Res 2005, 84:245-249.

22. Koga $H$, Ishibashi $H$, Shimada $H$, Jang IS, Nakamura TY, Nabekura J: Activation of presynaptic GABAa receptors increase spontaneous glutammate release onto noradrenergic neurons of the rat locus coeruleus. Brain Res 2005, 1046:24-31.

23. Xu Z-QD, Shi TJS, Hokfelt: Galanin-GMAP and NPY-like immunoreactivities in locus coeruleus and noradrenergic nerve terminals in the hippocampal formation and cortex with notes on the galanin-R1 and R2 receptors. J Comp Neurol 1998, 392:227-251.

24. Chan-Palay V: Galanin hyperinnervates surviving neurons of the human basal nucleus of Meynert in dementias on Alzheimer's and Parkinson's disease. A hypothesis for the role of galanin in accentuatine cholinergic dysfunction in dementia. J Comp Neurol 1988, 273:543.

25. Hoogendijk WJ, Feenstra MG, Botterblom MH, Gilhuis J, Sommer IE, Kamphorst W, Eikelenboom P, Swaab DF: Increased activity of surviving locus coeruleus neurons in Alzheimer's disease. Ann Neurol 1999, 45: 82-91.

26. Nieuwenhuys $R$, Geeraedts $L M$, Venning JC: The medial forebrain bundle of the rat. J Comp Neurol 1982, 206(1):82-108.

27. Batini C, Rossi GF, Zanchetti A, Moruzzi G: Brainstem reticular formation. Anatomy and Physiology. Arch Ital Biol 1995, 95:1-12.

28. van der Kooy D, Phillips AG: Involvement of the trigeminal motor system in brainstem self stimulation and stimulation-induced behavior. Brain Behav Evol 1979, 16:293-314.

29. Takamatsu J, Inoue T, Tsuruoka M, Suganuma T, Furuya R, Kawawa T: Involvement of reticular neurons located dorsal to the facial nucleus in activation of the jaw-closing muscle in rats. Brain Res 2005, 1055:93-102.

30. Scudder C, Kaneko CS, Fuchs A: The brainstem burst generator for saccadic eye movements: a modern synthesis. Exp Brain Res 2002, 142:439-462.

31. Baker R, Gresty M, Berthoz A: Neuronal activity in the prepositus hypoglossi nucleus correlated with vertical and horizontal eye movement in the cat. Brain Res 1977, 101:366-371.

32. Dean $P$, Mayhew $E$, Langdon P: Learning and maintaining saccade accuracy: a model of brainstem cerebellar interactions. J Cogn Neurosci 1994, 6:117-138.

33. Iwamoto Y, Yoshida K: Saccadic dysmetria following inactivation of the primate fastigial oculomotor region. Neurosci Lett 2002, 325:211-215.

doi:10.1186/1752-1947-6-161

Cite this article as: Cicco: Central syntropic effects elicited by trigeminal proprioceptive equilibrium in Alzheimer's disease: a case report. Journal of Medical Case Reports 2012 6:161. 